\title{
Féeries
}

Études sur le conte merveilleux, XVII $-\mathrm{XIX}{ }^{\mathrm{e}}$ siècle

13 | 2016

Contes et morale(s)

\section{Une réécriture russe des contes de Charles Perrault}

A Russian Rewriting of the Fairy Tales of Charles Perrault

\section{Véronika Altachina}

\section{(2) OpenEdition}

Journals

Édition électronique

URL : http://journals.openedition.org/feeries/1016

ISSN : 1957-7753

Éditeur

UGA Éditions/Université Grenoble Alpes

Édition imprimée

Date de publication : 15 octobre 2016

Pagination : 235-250

ISBN : 978-2-8310-335-3

ISSN : 1766-2842

\section{Référence électronique}

Véronika Altachina, «Une réécriture russe des contes de Charles Perrault », Féeries [En ligne], 13 |

2016, mis en ligne le 01 janvier 2017, consulté le 08 septembre 2020. URL : http://

journals.openedition.org/feeries/1016

\section{(c) Féeries}




\section{UNE RÉÉCRITURE RUSSE DES CONTES DE CHARLES PERRAULT}

$L$

es contes de Charles Perrault, traduits pour la première fois en I768, connurent une grande vogue en Russie après la traduction de 1867 ornée des gravures de Gustave Doré et accompagnée du nom d'Ivan Tourgueniev ${ }^{1}$ qui, en fait, n'a traduit que deux contes, "La Barbe bleue» et "Les Fées ${ }^{2}$. La fin du XIX siècle voit paraître de nombreuses nouvelles traductions et adaptations parmi lesquelles une qui tient une place particulière, celle d'Ekatérina Ursinovitch faite en $1897^{3}$ et rééditée quatre fois jusqu'en I9I5.

Ekatérina Vakchtérova (I867-1935), épouse Ursinovitch, était institutrice, écrivain pour les enfants, rédactrice de recueils de poésies et de récits pour les petits publiés régulièrement dans les années 1890-1900. Son frère aîné4 était un pédagogue et savant connu, didacticien de l'école primaire, auteur de plusieurs livres et manuels, fondateur de la pédagogie évolutive liée au concept de l'enseignement comme un des facteurs primordiaux du progrès social. Comme son frère, auteur, entre autres, de l'Abécédaire russe (I898) et du Livre de lecture pour les enfants (1902), Ekatérina Ursinovich était persuadée que la lecture joue un rôle très important dans l'enseignement et l'éducation. Deux fois dans sa vie, elle s'appliqua à la traduction de contes français : ceux de Perrault en I897 et de la comtesse de Ségur (I799-I874)

I. Ivan Tourgueniev (I818-1883), un des plus grands et des plus connus écrivains russes, romancier, novelliste et dramaturge. Son roman le plus célèbre est Pères et Fils (I862).

2. Перро Шарль. Волшебные сказки. Имлюстрации Г.Аоре. Перевод Ивана Тургенева. Москва: изА-во Маврикия Вольфа, I867, 76 с. - Ch. Perrault, Contes merveilleux. Illustrations de G. Doré. Traduction d'I. Tourgueniev, Moscou, éd. Mavrikii Wolf, 1867.

3. Перро. Сказки. Волшебный мир. Пер. Ек. Урсынович. С илмюстрациями Г.Аоре. Москва: Типография Высочайше утвержденного товарищества И.А.Сытина, I897, I43 c. - Ch. Perrault, Contes. Le Monde merveilleux. Avec les illustrations de G. Doré. Traduction d'E. Ursinovitch, Moscou, typographie de la compagnie de I.D. Sitine, 1897. Cette édition sera désormais citée dans les notes sous le nom d'Ursinovitch.

4. Vakchtérov Vassiliï Porfirièvich (I853-1924). 
en I9I3 dont elle traduisit le conte «Ourson» tiré des Nouveaux Contes de fées (1856). Elle était convaincue de la nécessité d'apprendre la lecture et l'écriture aux enfants et au peuple, et elle a déployé de grands efforts pour intégrer ces apprentissages dans son travail d'institutrice. En 1920 elle fut arrêtée et condamnée pour des raisons politiques. Elle ne fut réhabilitée qu'en 2004.

Pour Ursinovitch, très proche de l'organisation Narodnä̈a Volia à laquelle appartenait son mari $^{6}$ et dont le but était de libérer la population, la cause du peuple était très importante. La traductrice elle-même consacra sa vie à apprendre à lire et à écrire à des enfants et à des adultes illettrés, notamment en travaillant dans une école du dimanche à Vilna, dans une province du nord-ouest de la Russie.

Faute de renseignements exacts sur sa vie, il est difficile de dire quand, et pour quelles raisons, elle décida de faire une nouvelle traduction de Perrault. Non seulement la traduction dite de Tourgueniev avait été rééditée plusieurs fois, mais en 1896 deux autres traducteurs avaient encore proposé leurs propres variantes des contes. On peut toutefois supposer que sa traduction entrait dans le cadre de ses tâches éducatives et de son travail d'institutrice.

Ursinovitch choisit l'édition de Hetzel de I862 avec les gravures de Doré, édition déjà utilisée par Tourgueniev et, donc, connue du public. Mais au lieu de faire une édition de luxe avec reliure en cuir estampé d'or, tranche dorée, format in-folio avec des gravures en pleine page, tout en conservant les gravures, elle proposa un livre beaucoup plus modeste et moins cher destiné à l'apprentissage de la lecture aux enfants ou aux adultes avec des caractères appropriés, grands et bien lisibles. Elle respecte l'ordre des contes et garde les titres proposés dans l'édition de Tourgueniev à l'exception de «La belle endormie» ("La Belle au bois dormant ${ }^{7}$ ) qui devient «Le royaume du sommeil ${ }^{8}$ ». Je reviendrai sur ce titre plus loin.

5. Narodnaïa Volia (en russe : Народная воля; «Volonté du Peuple» ou «Liberté du Peuple») est une organisation anarchiste terroriste russe de la fin du XIX ${ }^{e}$ siècle, responsable de plusieurs attentats à la bombe, dont l'assassinat de l'empereur Alexandre II en I88I. Le programme du mouvement fut simple et se résuma à un seul but : «Substituer à la volonté despotique d'un seul la volonté du peuple».

6. Ursinovitch Léontiï Vladislavovich (1856-après 1926), étudiant de l'Université de SaintPétersbourg, participa à la préparation de l'attentat contre Alexandre II en I88I et fut envoyé en Sibérie en I882. Après 1917, il a collaboré à l'Association des condamnés politiques.

7. J'utiliserai ce titre français dans les citations pour ne pas perturber le lecteur par un titre inhabituel.

8. Toutes les traductions sont faites par l'auteur de l'article. 
Ses objectifs pédagogiques se manifestent dans de nombreuses modifications des contes de Perrault. D'une part, pour qu'ils soient bien compris par des petits lecteurs, elle les place dans la tradition des contes populaires et littéraires russes. D'autre part, prenant bien soin de l'utilité de la lecture et de la lucidité des leçons morales, elle réorganise le texte de sorte que son didactisme soit non seulement bien net, mais à la portée des enfants. Ces deux axes de transformation déterminent la structure de l'article : je commencerai par la russification des contes pour terminer par leur didactisme.

\section{La russification des contes}

Bien que les premiers recueils de contes russes soient apparus à la fin du XVIII ${ }^{\mathrm{e}}$ siècle", les contes folkloriques ne deviennent à la mode que dans la deuxième moitié du XIX ${ }^{\mathrm{e}}$ siècle grâce à la publication des Contes populaires russes (Narodnye russkie skazki), entre I855 et I863, par Alexandre Afanassiev (I826-I87I), folkloriste, collecteur de contes du folklore et éditeur. Le succès du recueil fut immense et immédiat en Russie et ne s'est jamais démenti. Il en existe plusieurs éditions complètes et annotées, et d'autres pour les enfants. Les contes furent illustrés par Viktor Vasnetsov ${ }^{\text {10 }}$ et Ivan Bilibine ${ }^{\text {II }}$ qui ont rendu les sujets folkloriques encore plus attrayants et célèbres. Les contes d'Afanassiev proviennent d'archives, de collections déjà constituées, comme celle de Vladimir Dahl (I80I-I872), lexicographe et écrivain russe, auteur du fameux Dictionnaire raisonné du russe vivant, paru en quatre tomes entre I863 et I866 (réédité cent fois de 2005 à 20I5) qui, en I832, publia les Contes et légendes russes (interdits par la censure) et, entre 1833 et I839, Histoires véritables et imaginaires. Ses œuvres

9. D. Tchoulkov, Persifleur ou les Contes slaves (1766-1768); Les contes de ma grand-mère (1778); Chouette, oiseau de nuit (1779); V. Levchin, Les Contes russes (1780-1783). Catherine II écrivit deux contes pour les enfants, Tzarévitch Chklore (I78I) et Tzarévitch Févéï (I782).

Io. Viktor Vasnetsov (I848-1926) est un artiste russe qui se spécialisa dans les représentations mythologiques et historiques. Il est considéré comme l'un des peintres les plus influents de l'art russe de la fin du XIX et du début du Xxe siècle. À la fin des années i870, Vasnetsov se consacre à l'illustration des contes russes et commence à travailler à son Tsarévitch Ivan montant un loup gris et à L'Oiseau de feu.

II. Ivan Bilibine (I876-1942) est un peintre russe, illustrateur de livres et décorateur de théâtre, membre de l'association Le Monde de l'Art (Mir iskousstva / Мир искусства). Les six fascicules de contes publiés par le Goznak de I90I à I903 ont paru en français en I98I, avec la traduction de 1976 de Luda et les illustrations originales de Bilibine, imprimés par le Goznak à Moscou pour les Éditions La Farandole, sous les titres suivants : L'Oiseau de feu — conte d'Ivan-tsarévitch, de l'oiseau de feu et du loup gris; La Princesse-Grenouille; Vassilissa-la-très-belle; La Plume de Finist -Fier Faucon; Maria des Mers; Grande-Saur et Petit-Frère; Blanche Canette. 
complètes furent éditées deux fois, en I86I et en I883-1884, par Mavriki Wolf, celui qui publia également la traduction des contes de Perrault par Tourgueniev. Afanassiev suscite l'intérêt pour le conte comme le feront à leur tour Evguéni Tchoudinski dans ses Contes, quolibets et fables (I864), puis Dimitri Sadovnikov dans Contes et légendes de la région de Samara (I884). Il ne faut pas non plus oublier la tradition littéraire représentée par Alexandre Pouchkine (I799-I837) dont les contes en vers furent publiés entre I830 et I834, les contes poétiques (I832-I845) de Vassili Joukovski (1783-1852) et Le Petit cheval bossu (I834) de Pavel Erchov (I815-1859).

Cette vogue du folklore russe correspondant à l'intérêt pour le peuple de Narodnaïa volia s'associe à la redécouverte des contes de Perrault à la suite de l'édition de Tourgueniev qui poussa Ursinovitch à faire fusionner le contenu français avec l'esprit russe pour aboutir à une création originale, sans précédents ni successeurs. La russification des contes de Perrault dans la traduction d'Ursinovitch se fait à quatre niveaux : au niveau du lexique, des proverbes et dictons, des personnages récurrents et, finalement, des expressions et phrases rituelles.

\section{Le lexique}

L’objectif pédagogique d'Ursinovitch détermine le choix de son lexique et le style de sa traduction : pour que les contes français soient bien compris, elle procède à leur stylisation à la façon des contes populaires russes. Premièrement, elle remplace tous les éléments de la vie française par des équivalents russes. Par exemple, le seigneur ne rembourse pas sa dette de Io écus, mais le bûcheron trouve une bourse pleine d'argent. De même, au lieu d' "un étui d'or massif» avec "une cuillère, une fourchette et un couteau d'or fin, garni de diamant et de rubis ", inconcevables pour un petit lecteur du peuple ${ }^{\mathrm{r} 2}$, on retrouve une simple "assiette en or $^{13}$ » qui est en réalité tirée du conte des frères Grimm et déjà utilisée par un poète russe très connu, Vassili Joukovski, dans sa variante de "La Belle au bois dormant» (I832). Quant au «royaume de Mataquin, situé à douze mille lieues» il est, bien sûr, omis. Dans "Le Chat botté», le lion et l'éléphant dont l'aspect peut surprendre ${ }^{\mathrm{I} 4}$ sont remplacés par des animaux connus des petits Russes, un âne et un $\operatorname{cochon}^{15}$.

I2. Ch. Perrault, Contes, Paris, Librairie générale française, 2006, p. 186.

I3. Ursinovitch, p. 4r.

I4. Ch. Perrault, ouvr. cité, 2006, p. 24I.

15. Ursinovitch, p. 82. 
Étant donné son caractère encyclopédique, le conte populaire touche ainsi à presque toutes les sphères de la vie sociale. Pour reprendre les mots de Christina Kossogorova, on peut dire que "[le] noyau principal du lexique des termes sans équivalents contient les mots et les groupes de mots désignant des objets spécifiques au mode de vie, à la culture, au degré de développement social et historique d'un peuple. Ces termes, en raison de leur appartenance à une sémantique nationale très marquée quel que soit le niveau de la langue dont ils relèvent ${ }^{16}{ }^{\prime}$, donnent ainsi aux contes une couleur locale très intense qu'Ursinovitch cherche à recréer en remplaçant la réalité française par le lexique de la vie quotidienne russe : "izba», «khatka» pour «maison", "pirojok» pour "galette», les bottes de sept lieues deviennent celles de "sept verstes ${ }^{17}$ ", le bûcheron amène les enfants dans la forêt pour "ramasser les champignons", les enfants mangent "de la bouillie» et le froid gèle les rivières.

La traductrice emploie également le langage parlé des contes populaires avec beaucoup de diminutifs, de mots familiers (saloperie, gueule, pauvre idiot, bonnes gens, canaille, gosse, gars, bougre, mémère, taudis, cabane, etc.), d'expressions idiomatiques dont il est difficile de trouver des équivalents en français (rire et pleurer ${ }^{18}$, aller à l'aventure ${ }^{\mathrm{r} 9}$, croquer à belles dents - rendre la vie impossible à quelqu'un ${ }^{20}$, être à portée de main ${ }^{21}$, par monts et par vaux ${ }^{22}$, n'avoir qu'une idée en tête ${ }^{23}$, il n'est pas né d'hier ${ }^{24}$, réduire en poussière ${ }^{25}$, etc.), de comparaisons ou épithètes figées («les cheveux de lin», «les yeux de myosotis» dans la description du Petit Chaperon rouge ${ }^{26}$, «les lèvres de miel », « une belle aux cheveux dorés ${ }^{27}$ ). On retrouve des épithètes récurrentes : "la sombre forêt ${ }^{28}$ ", «l'épaisse forêt ${ }^{29}$ » — lieu d'initiation, d'épreuve pour le Petit Poucet, ainsi que pour

I6. C. Kossogorova, "Aspects sociolinguistiques et valeur culturelle des contes russes», dans la Revue du Centre Européen d'Études Slaves, 20II, n I, n. p., accessible sur http://etudesslaves.edel. univ-poitiers.fr/index.php?id=76 (date de consultation : oI/o2/20I6).

17. Mesure itinéraire ancienne valant $1067 \mathrm{~m}$.

18. Ursinovitch, p. I6.

19. Ibid., p. 35 .

20. Ibid., p. I24.

2I. Ibid., p. 36 .

22. Ibid.

23. Ibid., p. 20.

24. Ibid., p. 73.

25. Ibid., p. 78 .

26. Ibid., p. 5.

27. Ibid., p. 55 .

28. Ibid., p. 5I-52.

29. Ibid., p. 20 et 27. 
le Prince dans «La Belle au bois dormant». Cette idée est mise en relief dans le texte russe : si, au début, le Petit Poucet marche le dernier, c'est lui qui se met en tête pour ramener ses frères, et c'est lui qui devient le héros national applaudi par la foule criant : «Vive le Petit Poucet ${ }^{30}$ !" Quant au prince, il décide soit de sauver la belle princesse, soit de périr, et il traverse sans hésiter la forêt épaisse, franchit des ravins profonds et des pentes raides $^{31}$. Le compère loup du "Petit Chaperon rouge" devient le loup gris, personnage traditionnel des contes populaires ${ }^{32}$.

En suivant le modèle des contes russes, la traductrice a également recours aux répétitions de renforcement : "en chemin - en route ${ }^{33}$ ", "pleurer larmoyer ${ }^{34}$ ", "embrasser - caresser ${ }^{35}$ ", " réfléchir profondément à une pensée ${ }^{36} »$. Elle a également recours à des rimes et des assonances, "de retour dans leur demeure, ils mangent de la bouillie avec du beurre ${ }^{37}$ », "un chaton au front grison ${ }^{38}$ ".

La traductrice s'adresse ainsi aux enfants en facilitant leur compréhension par un vocabulaire familier, et fait appel à la couleur locale pour transposer les contes français dans la culture russe qui est la leur.

\section{Proverbes et dictons}

Depuis la publication du Dictionnaire de Dahl ${ }^{39}$, les proverbes et les dictons sont considérés comme une partie très importante de la langue russe. De plus, ils étaient insérés dans les abécédaires. Ces expressions figées n'ont pas d'équivalents en langue étrangère, mais «ce sont ces unités langagières qui donnent à la langue russe sa vivacité et sa tonalité si particulière. Elles sont aussi une ressource inépuisable pour qui veut connaître la culture, l'histoire et de la psychologie du peuple russe ${ }^{40}$ ». Les proverbes résument la morale des contes pour la rendre plus accessible à la mentalité russe. Deux contes se terminent ainsi sur des proverbes russes : «dans les petits

30. Ibid., p. 40 .

31. Ibid., p. 52.

32. Ibid., p. 5 .

33. Ibid., p. 108.

34. Ibid., p. I9.

35. Ibid., p. 2I.

36. Ibid., p. 20.

37. Ibid., p. 22.

38. Ibid., p. 73.

39. Ааль В.И. Толковый словарь живаго великорусского языка. - V. Dahl, Dictionnaire raisonné du russe vivant, accessible sur http://slovardalja.net/ (date de consultation : oI/O2/2016).

40. C. Kossogorova, art. cité, n. p. 
pots les bons onguents» pour «Le Petit Poucet», et «il file des jours d'or et de soie» pour "Le Chat botté». La traductrice cite également des proverbes courants : "dans la vie faut pas s'en faire ${ }^{41}$ " ou "avoir une grande ouverture d'esprit ${ }^{42}$ ", "plus on a d'esprit, plus on croit en manquer ${ }^{43}$ ". Les proverbes résument la sagesse du peuple, rendant la morale plus générale tout en renforçant la couleur locale.

\section{Personnages récurrents}

La russification concerne également les personnages traditionnels des contes de fées, notamment la fée, mot qui existait en russe, mais qui n'entra dans le vocabulaire qu'au début du XIX ${ }^{\mathrm{e}}$ siècle. La première mention de "Fée" dans le dictionnaire date de I837. Elle n’avait donc pas été utilisée dans les contes populaires sur lesquels Ursinovitch s'était appuyée pour sa traduction. Ursinovitch avait cependant trouvé des synonymes différents correspondant au contexte : la mauvaise fée de "La Belle au bois dormant» devient sorcière, tandis que les autres au nombre de sept - le sept est souvent utilisé dans le folklore russe ${ }^{44}$ — sont des enchanteresses ou des magiciennes, comme la marraine de Cendrillon et le personnage éponyme des "Fées». L'enchanteresse (en russe «чародейка», celle qui fait les charmes) apparut dans la langue russe au XI ${ }^{\mathrm{e}}$ siècle. Elle était souvent employée dans la poésie lyrique du XIX ${ }^{\mathrm{e}}$ siècle : on peut citer les noms de grands poètes russes de l'époque comme Derjavine, Batiouchkov, Baratinski, Joukovski, notamment dans son poème "La belle au bois dormant" (I832) où il réunit les contes français et allemands. Mais ce mot devient surtout à la mode après le grand succès de l'opéra de Piotr Tchaïkovski, L'Enchanteresse, créé au théâtre Mariinski de Saint-Pétersbourg en I887. Quant à «la magicienne", elle existait déjà dans le slavon et fut largement nommée dans les contes populaires et littéraires. On peut noter que la première traduction des contes de Perrault fut sous-titrée : Les contes sur les magiciennes avec les moralités $(\mathrm{I} 768)^{45}$.

4I. Ursinovitch, p. 70.

42. Ibid., p. 88.

43. Ibid., p. 92.

44. On peut juste nommer les contes les plus connus : le conte populaire des Sept frères Sémion (I786) ou le conte en vers de Alexandre Pouchkine La princesse morte et sept bogatures (héros des épopées russes) (1833).

45. Перро Ш. Скаски о волшебницахъ съ нравоученїями. Переведены съ французскаго [Аьвом Воиновым]. Печатаны при Императорскомъ Московскомъ Университетњ, I768, I40 c. - Ch. Perrault, Contes sur les magiciennes avec les moralités. Traduits du français par L. Voïnov, Moscou, Université de Moscou, 1768. 
«L'ogre» dans les premières traductions russes fut traduit par «sauvage, sylvain ". La traduction exacte de ce mot dans les dictionnaires françaisrusses de l'époque est "sylvain, anthropophage ${ }^{46}$ ». "Ludoed», l'ogre en russe, est le calque de "l'anthropophage». Si dans «Le Petit Poucet» il mérite bien son nom et veut manger les petits enfants, dans «Le Chat botté» ce n'est pas le cas et la traductrice le nomme "Géant - Gros mangeur" - personnage qui existait déjà dans un conte russe, Le bateau volant.

Annouchka et Alionouchka des "Fées» sont deux prénoms traditionnels des contes russes, de même le maître du «Chat botté» est nommé Ivan-tzarévitch, troisième fils de famille, surnommé Ivan le Nigaud (Ivan l'Idiot), héros de plusieurs contes dont le plus connu est Ivan tzarévitch et le loup gris. Si, au début des contes, il n'a pas de chance et est méprisé, c'est lui qui, à la fin, épouse une belle tzarevna (princesse) et devient roi, tout comme dans le conte français. Dans le conte cité ci-dessus, c'est le loup - le chat chez Perrault — qui l'aide à réussir. Mais le chat, lui aussi, revêt, dans la traduction, des traits du personnage des contes littéraires russes inspirés par le folklore : il est nommé le "chaton au front grison" - personnage du conte du pédagogue connu Konstantin Ouchinski (I824-I87I), Le chat coquin (1864) - ou "Chaton Ronron" - personnage de La guerre des souris et des grenouilles (I832) de Joukovski, ainsi que du recueil Les contes du Chaton Ronron (1872) de Nicolas Wagner (I829-1907).

Ursinovitch traduit le titre du conte «Riquet à la houppe»par «Le toupet», qui a déjà été employé par Tourgueniev : le nom propre Riquet n'est même pas cité. Ce titre vient d'un conte populaire russe, dans lequel il figure une sorte de diable. Dans le Dictionnaire de Dahl, «le toupet» ("khokhlik» en russe) est présenté comme "un diable, un démon», la houppe servant de substitut pour les cornes. Le festin qui se prépare sous terre, ainsi que la laideur et l'intelligence justifient en quelque sorte cette allusion. Le Dictionnaire des synonymes de la langue russe propose comme synonyme "un être imaginaire ${ }^{47}$ ». Le mot fut souvent utilisé dans des textes de l'époque, tantôt comme diminutif pour un Ukrainien à cause

46. Международные словари дмя средних учебных заведений. Часть французско-русская. Н.Макаров. СПб.: типография Тренке и Фюсно, І893. - Dictionnaire français-russe, par N. Makarov, Saint-Pétersbourg, typographie Trenke te Fusneau, I893.

47. Словарь синонимов русского языка. - Dictionnaire des synonymes de la langue russe, n. p., accessible sur http://endic.ru/synonym/Hohlik-34064.html (date de consultation : oI/o2/2016). 
de sa coiffure traditionnelle, tantôt comme synonyme pour un gars, un bonhomme ${ }^{48}$.

Ainsi, en recourant aux personnages bien connus des enfants ainsi que des adultes, la traductrice facilite la compréhension des contes : les nouveaux personnages pourvus de noms et de traits traditionnels sont perçus comme faisant partie des leurs.

\section{Expressions et phrases rituelles}

La longue tradition des contes populaires — oraux ou écrits - a consacré certaines formules, surtout pour l'incipit. C'est Charles Perrault qui introduit dans le conte français la fameuse expression "Il était une fois» : il l'emploie pour la première fois dans "Peau d'âne» en I694, et commence par elle sept de ses contes en prose à l'exception du "Chat botté». En russe il existe une formule équivalente, mais Ursinovitch ne l'utilise pas : tous ses incipit sont différents, mais tous font référence au folklore.

Deux contes débutent par la même phrase rituelle : «Dans un lointain royaume, dans un pays lointain ». Les deux contes traitent d'une jeune fille humiliée et malpropre, d'une salisson — les mêmes épithètes caractérisent les deux hérö̈nes devenues reines : "Cendrillon» et "Peau d'âne». Une formule semblable est utilisée au début de "La Belle au bois dormant»: "Par-delà la trois fois neuvième terre, dans le trois fois neuvième royaume", c'est-à-dire "dans un pays très lointain, dans un royaume très lointain". Ces incipit reviennent dans plusieurs contes merveilleux et plongent le lecteur dans une ambiance magique. Pour "Les Fées», la traductrice a recours au début à un conte en vers très connu d'Erchov, Le Petit cheval bossu: "Au-delà des monts, des ondes, au-delà des mers profondes». Les autres incipit servent à focaliser l'attention du lecteur sur le sujet principal : "Une paysanne avait une fille de six ans si mignonne que tous l'admiraient» ("Le Petit Chaperon rouge»), "Dans une pauvre masure près de la forêt il était une fois un bûcheron avec sa femme» («Le Petit Poucet»), «Une fois, il y a très longtemps, trois fils du meunier décédé se mirent à partager leur héritage» ("Le Chat botté»), «Il y avait un grand festin dans un palais de marbre» ("Riquet à la houppe»), ou encore "Très loin d'ici un homme bien riche surnommé La Barbe bleue habitait dans un palais superbe» («La Barbe bleue»). Dans «Peau d'âne» on retrouve la

48. Национальный корпус русского языка - Le corpus national de la langue russe, n. p., accessible sur http://ruscorpora.ru/search-main.html (date de consultation : oI/o2/20I6). 
formule finale des contes populaire russes : «La fin du conte est pour vous, et un pot de miel - pour moi.»

Dans les contes on retrouve d'autres expressions traditionnelles grâce auxquelles les petits lecteurs se retrouvent dans un monde connu, perçoivent le conte étranger comme le leur, ce qui favorise leur compréhension. Ce sont des formules d'indétermination de temps et de lieu, propres au conte merveilleux : "(Chemina-t-il) longtemps ou non, près ou loin, haut ou bas ${ }^{49}$ ", "conter, c'est vite fait, agir, c'est bien plus long ${ }^{50}$ ", ou «la bouche ne peut le dire, la plume ne peut le décrire ${ }^{5 \mathrm{I}}$, clichés utilisés pour parler de quelque chose ou de quelqu'un d'une grande beauté, généralement une princesse.

Mais si la traductrice puise en abondance dans le folklore russe, elle s'adresse également à la tradition littéraire, comme je l'ai déjà noté, en citant des écrivains russes. Dans ce contexte, c'est le titre «Le Royaume du sommeil» au lieu de «La Belle endormie», entré désormais dans l'usage, qui attire notre attention. Le titre choisi par Ursinovitch est emprunté au poème de Mirra Lokhvitskaya (I869-1905), «Une fleur d'eau» (I894), où la description poétique et énigmatique du «royaume du sommeil», faite avec grande sensibilité et sensualité, nous rappelle la forêt traversée par le prince :

L'ombre tremblante des arbres...

Demi-lumière, silence...

La journée chaude ne pénètre pas

À travers la forêt épaisse dans le royaume du sommeil ${ }^{52}$.

Une belle jeune fille sort des nymphéas toute pâle, confuse. Ses traits, voilés par le mystère et l'attente, ses mouvements exprimant la langueur et la paresse, promettent "le nirvana des plaisirs", non pas grâce aux charmes de la beauté, mais par "l'éternité de l'oubli». Dans les années I890, Lokhvitskaya fut l'une des plus célèbres poétesses de l'époque, ses poèmes furent édités dans de nombreuses revues, et son premier recueil, sorti en I896, connut un immense succès et reçut, une année plus tard, le prestigieux Prix Pouchkine.

L'incipit de ce conte, déjà cité, peut se traduire littéralement par «Pardelà la trois fois neuvième terre, dans le trois fois neuvième royaume " qui

49. Ursinovitch, p. Io8.

5o. Ibid.

5I. Ibid., p. 98.

52. Аохвицкая Мирра. Водяной цветок. - M. Lokhvitskaya, La fleur de l'eau, n. p., accessible sur http://libverse.ru/lohvickaya/vodianoi-cvetok.html (date de consultation : 2I/02/2016). 
désigne, dans les contes russes, un royaume très lointain où le héros va trouver des objets magiques : les pommes qui rendent la jeunesse, des animaux (cochon ou cheval) tout en or, ou l'eau vive qui ranime les morts, ou encore une belle princesse. Selon Propp, c'est un royaume qui est «autre», "extraordinaire», séparé du monde réel par une forêt épaisse, une mer, une rivière de feu, une montagne ou un précipice et il n'y a qu'un seul bâtiment, un magnifique palais. Tout, dans ce royaume, est lié à l'or ou à la lumière. Nous retrouvons tous ces éléments chez Ursinovitch : le royaume du sommeil est ensorcelé, entouré d'une forêt impénétrable, séparé par des ravins profonds, par des pentes raides, le jet d'eau se fige tout en "étincelant», partout abondent «des fontaines d'argent, des miroirs, l'éclat et l'or». Les magiciennes déposent la princesse endormie, «belle comme le matin de mai ", donc forcément lumineuse, dans «la salle du palais en cristal» (qui brille) sur un lit en marbre ${ }^{53}$. La belle princesse a "les cheveux d'un blond doré», et son sourire est "clair» ${ }^{54}$. Tout est immobilisé par le sommeil dans ce "royaume des morts ${ }^{55}$ " - encore une expression figée, répétée à trois reprises, pour évoquer le lieu magique des contes russes qui, dans le folklore, est souvent lié à l'épreuve du sommeil.

Quand la princesse voit la vieille qui file, c'est la méchante sorcière, précise Ursinovitch, celle-ci chante une chanson dont le texte est tiré du conte en vers de Joukovski "La Belle au bois dormant" (I832) où le poète conjugue le conte de Perrault avec celui des frères Grimm (I8I2), traduit par lui-même en I826. D'autres références à ce conte russe connu apparaissent dans le texte d'Ursinovitch : les assiettes d'or au baptême de la princesse, les enchanteresses au lieu des fées, l'interdiction de cultiver le lin dans le royaume, l'absence d'oiseaux et de nuages dans le ciel pour décrire le royaume endormi où, avec leur fille, se retrouvent le tzar et la tzarine.

Si Ursinovitch adapte son lexique et ses références à un jeune public, les nombreuses références qu'elle fait à la poésie russe s'adressent plutôt à un public adulte et lettré. Ainsi, la description de la princesse dans «Peau d'âne» emprunte aux citations de "Olaf et Estrilde» (I886), une ballade du poète russe Sémion Nadson (I862-I887) qui a connu une extraordinaire popularité renforcée par sa mort précoce. Son premier recueil, publié en I885, eut un énorme succès et fut réédité vingt-neuf fois jusqu'en I9I7, mais sa gloire atteignit son apogée après les publications posthumes. La traductrice cite presque littéralement la description de la princesse Estrilde pour

53. Ursinovitch, p. 5I-52.

54. Ibid., p. 55-56.

55. Ibid., p. 5I-52. 
décrire Peau d'âne : elle est mince et svelte «comme un sapin vert dans des forêts vierges", blanche comme "la neige étincelant sur les hautes montagnes», belle "comme un matin de mai " ${ }^{56}$. Sa parure est "plus éclatante que les cieux printaniers", "ses cheveux d'un blond doré sont en boucles" sur ses épaules, et "ses yeux sont bleus comme la mer profonde».

Si le petit lecteur peut se rappeler certains vers de Joukovski et d'Erchov ou les personnages connus tels que Ivan-tzarévitch, le Chaton Ronron ou le Gros mangeur, c'est à l'adulte d'apprécier toute la richesse des références littéraires : les contes d'Ursinovitch s'adressent aux petits et aux grands, aux enfants et aux parents comme le pratiquait l'écrivain français.

\section{Le didactisme}

Passons maintenant aux raisons didactiques qui ont également déterminé les objectifs de la traductrice. Celle-ci modifie le texte de Perrault s'il ne répond pas, à son avis, à ses propres tâches éducatives ou s'il peut choquer l'imagination des enfants. Tout d'abord, elle omet toutes les scènes féroces et les images lugubres : la description très colorée des filles de l'Ogre ou les hurlements des loups dans la forêt dans "Le Petit Poucet», le Petit Chaperon rouge est si agile que le loup ne réussit pas à l'avaler — elle saute du lit et crie si fort qu'un bûcheron arrive et tue le méchant loup, et «La Belle au bois dormant» se termine par le mariage. Parallèlement, elle refuse tout ce qui ne peut pas servir de bon exemple. Le Petit Poucet n'utilise les bottes de sept lieues que pour de bonnes actions. Il ne va pas voler la femme de l'Ogre, ne sert pas de courrier galant, mais va au champ de bataille et devient un héros de la patrie, félicité par le roi. Dans «Les Fées», le prince décide d'épouser la jeune fille non pour les richesses qu'elle peut lui apporter, mais sur les conseils du bon magicien.

Ursinovitch change également tout ce qui peut paraître injuste, ou choquant pour les petits : la sœur jumelle dans «Riquet à la houppe», oubliée par Perrault presque dès le début du conte, trouve son fiancé bien avant la belle princesse, le Petit Poucet n'est plus "le souffre-douleurs de la famille», ni Pierre, le fils préféré de sa mère. La veuve des «Fées» adore sa fille et déteste sa belle-fille, les parents du Petit Poucet trouvent une bourse pleine d'argent et achètent bien des provisions, mais ils n'ont aucun appétit sans leurs enfants et ne peuvent pas même avaler une bouchée. Enfin, tous les

56. Ibid., p. 100. 
méchants sont punis même si la traductrice tâche d'éviter la mort : seuls le loup et la Barbe bleue — un être presque inhumain — sont tués alors que la méchante sœur des "Fées» est bien bannie avec sa mère du royaume, mais elle ne meurt pas. Quelques-uns des personnages méchants ont des remords : les sœurs de Cendrillon ont honte de leur conduite envers elle.

Ursinovitch transforme également tout ce qui ne paraît pas logique : le singulier remplace ainsi le pluriel dans le titre des "Fées" puisqu'il s'agit d'une même fée. Il serait difficile d'expliquer ce pluriel aux enfants, pluriel que ne justifie plus le texte de Perrault, contrairement à d'autres versions, dont celle de Basile, où les fées sont en effet au nombre de trois. Dans le même conte, la mère ne chasse pas sa fille qui lui apporte tant de trésors mais, devant la colère de sa belle-mère, c'est la jeune fille qui prend la fuite. Dans «Le petit Chaperon rouge», la grand-mère n'est pas malade, sa petite-fille lui porte des cadeaux pour sa fête. Si elle était malade, ce serait alors à sa fille d'aller la voir pour la soigner. Dans "Le petit Poucet", la femme de l'ogre, si bonne et charitable, ne peut pas être la mère des petites ogresses, portraits crachés de leur père, qui vont jusqu'à mordre leur bonne belle-mère ${ }^{57}$.

La traductrice ne conserve pas les moralités poétiques, mais elle les rend en prose tout en n'en gardant que ce qui lui paraît utile aux jeunes lecteurs. L'absence des moralités s'explique par la fidélité à l'édition d'Hetzel, ou même par l'usage dans les traductions russes du XIX ${ }^{\mathrm{e}}$ siècle, mais aussi par l'objectif pédagogique d'Ursinovitch, étant donné que les moralités de Perrault ne sont pas destinées à un jeune public. De ce fait, la traductrice omet tout ce qui n'est pas destiné aux oreilles des petits et crée des doubles sens chez Perrault. Sa morale bien claire est nettement exprimée dans le texte où la traductrice change les accents et renforce le didactisme voilé des contes français. Il n'y a que deux contes qui gardent exactement la moralité de Perrault, «Riquet à la houppe» et «Les Fées» où la traductrice insiste pourtant plus sur la punition des méchants que sur la récompense de la bonté.

Dans «Le Petit chaperon rouge», le mal est puni et le bien triomphe; le Petit Poucet fait non seulement le bonheur de sa famille mais il devient un héros de sa patrie; à la fin de "La Belle au bois dormant», la méchante fée disparait et dans le royaume il n'y a plus de malheur depuis, mais uniquement paix et amour. Cendrillon devient la protectrice de tous les enfants opprimés et ses sœurs rougissent de leur méchanceté envers elle. «Le Chat

57. Ibid., p. 32 . 
botté» et «Peau d'âne» refusent toute leçon moralisatrice et se terminent par un mariage heureux, traditionnel dans les contes russes. L'histoire macabre de «La Barbe bleue» n' est qu'un rêve, un cauchemar qu'on oublie rapidement.

Ursinovitch prône la bonté, la charité, la miséricorde et n’hésite pas à rajouter sa propre morale dans la traduction. Ainsi, parmi les dons des fées on voit la beauté, l'esprit et des talents artistiques, mais aussi le bon cœur, l'amour et la pitié pour les malheureux ${ }^{58}$. Sa marraine dit à Cendrillon que son "cœur doux et affectueux est beaucoup plus cher que l'or ${ }^{59}$ " et, quand ses sœurs lui demandent pardon pour leur comportement, elle leur répond par un proverbe: "Qui évoque le passé s'en repent.» Devenue reine, Cendrillon n'oublie pas qu'auparavant elle n'était qu'une pauvre enfant abandonnée et elle prend soin de toutes les "salissons" (c'est le titre du conte russe en usage depuis I825, repris dans l'édition de Tourgueniev, la traduction mot à mot qui est d'usage de nos jours n'apparaissant qu'en I875 et n'étant alors pas encore bien ancrée dans la tradition). Dans "Riquet à la houppe", la Princesse comprend qu'elle aime Riquet avant qu'il ne soit devenu beau. Ursinovitch choisit ainsi la deuxième explication de la métamorphose proposée par Perrault où tous les défauts de Riquet se transforment en qualités sous l'influence de l'amour : "C'est l'amour qui a fait ce miracle ${ }^{60}$.» Quand la Reine voit Peau d'âne dans toute sa beauté, elle dit, les larmes aux yeux, qu'elle avait toujours pensé que seuls les riches et les nobles étaient des gens bien, mais que, maintenant, elle voit que, sous la peau d'un âne sale, peut se cacher un cour généreux ${ }^{61}$.

Traduire, selon l'expression d'Umberto Eco, c'est dire presque la même chose et c'est ce "presque" qui assure l'adoption d'une œuvre par une autre culture. «Le transfert d'un texte dans une autre langue par le biais de la traduction implique également le transfert dans un autre type de généricité», écrit Ute Heidmann en appelant ce transfert «la (re)configuration générique $^{62} »$. Comme Perrault lui-même avait reconfiguré «les formes génériques du conte déjà existantes ${ }^{63}{ }^{\prime}$, Ursinovitch a, dans sa traduction,

58. Ibid., p. 42.

59. Ibid., p. 6I.

6o. Ibid., p. 96.

6I. Ibid., p. I2I.

62. U. Heidmann, «C'est par la différence que fonctionne la relation avec un grand R. Pour une approche comparative et différentielle du traduire», dans G. Chiurazzi (éd.), The Frontiers of the Other. Ethics and Politics of Translation, Philosophie - Sprache - Literatur 4, Lit. Verlag, Wien; Zürich; Berlin; Münster, 2013, p. 66.

63. Ibid., p. 67. 
elle-même reconfiguré les contes de Perrault en les insérant dans la tradition populaire et littéraire russe, en ayant recours à d'autres intertextes, comme l'avait fait Perrault. Les trois éléments de base des contes français sont sauvegardés dans la traduction : la composante folklorique, les allusions littéraires, les détails de la vie contemporaine.

La traduction d'Ursinovitch, une de ces «belles infidèles» où le mérite littéraire l'emporte sur la fidélité à l'original, reste paradoxalement très fidèle à l'esprit des contes de Perrault. "Quand la traduction est en prose et qu'elle a été faite par un habile homme, je soutiens qu'on y voit aussi bien les sentiments et les pensées de l'auteur comme dans ses propres paroles ${ }^{64}$.» Cette idée de Perrault correspond parfaitement bien à la traduction russe d'Ursinovitch.

64. Ch. Perrault, Parallèle des anciens et des modernes, 3 vol., Paris, Jean-Baptiste Coignard, I693, vol. 2, p. 5 . 
